\title{
Late Postoperative Rhinological Complications After Microscopic Transnasal Hypophysectomy
}

\author{
Lutfi POSTALCI ${ }^{1}$, Ibrahim ERDIM²${ }^{2}$, Bulent DEMIRGIL ${ }^{1}$, Omur GUNALDI ${ }^{1}$, Murad ASILTURK ${ }^{1}$, Hakan DEMIRCI ${ }^{1}$, \\ Hakan KINA ${ }^{1}$, Uzay ERDOGAN ${ }^{1}$, Mine $\mathrm{YAZICl}^{3}$, Erhan EMEL ${ }^{1}$ \\ 1Bakirkoy Research and Training Hospital for Neurology, Psychiatry and Neurosurgery, Neurosurgery Clinic, Istanbul, Turkey \\ ${ }^{2}$ Erbaa State Hospital, Otorhinolaryngology Clinic, Tokat, Turkey \\ ${ }^{3}$ Bakirkoy Dr. Sadi Konuk Training and Research Hospital, Otorhinolaryngology Clinic, Istanbul, Turkey
}

\section{ABSTRACT}

AIM: Major complications of microscopic transnasal hypophyseal surgery (MTHS), such as cerebrospinal fluid rhinorrhea, carotid injury, and optic nerve injury, are very rare. However, late rhinological complications can be ignored because they are a minor cause of morbidity compared with major complications. In this study, we extensively examined postoperative rhinological complications in patients who underwent MTHS for pituitary adenoma.

MATERIAL and METHODS: Thirty-one patients diagnosed with pituitary adenoma, who underwent MTHS and whose preoperative nasal examinations were recorded between January 2007 and January 2014, were included in the study. A detailed rhinological examination of the patients was performed.

RESULTS: A total of 12 of 31 patients (38.7\%) had a perforated nasal septum, and synechiae were detected in the nasal cavities of 13 patients (42\%). Anosmia occurred in three patients, hyposmia in two, and a nasal tip deflection and saddle nose deformity were detected in one patient with a perforated nasal septum. No perinasal loss of sense, oronasal fistula, or purulent secretion in the nasal cavity was found in any patient.

CONCLUSION: The nasal structures, particularly the nasal septum mucosa, should be treated gently during MTHS. The nasal stages of the operation should be performed with the help of an otolaryngologist until adequate experience is gained.

KEYWORDS: Transsphenoidal surgery, Nasal synechiae, Perforation, Hyposmia, Complication

\section{INTRODUCTION}

$\mathrm{T}$ The sphenoid sinus approach to the pituitary gland was a breakthrough treatment for lesions in this region. The lateral rhinotomy approach to trans-sphenoidal hypophysectomy was performed for the first time in 1907 by Schloffer (26). Halsted (9) and Hardy (10) laid the foundation for today's microscopic transnasal hypophyseal surgery (MTHS) procedure by improving their technique. The development of neuroendoscopy in the 1990s resulted in new approaches to this region (2).
Rhinological complications have been reported previously, but no study has thoroughly investigated them. In this study, we extensively examined postoperative rhinological complications in patients who underwent MTHS due to pituitary adenoma.

Serious vascular complications, such as carotid injury, as well as major complications, such as optic nerve injury and cerebrospinal fluid rhinorrhea, can occur during MTHS. However, rhinological complications can be ignored because they are a minor cause of morbidity compared to major complications. These patients may experience problems such as epistaxis, 
nasal crusting, or respiratory and olfactory disorders. Some of these complaints may require otolaryngological surgery.

\section{MATERIAL and METHODS}

Thirty-one patients diagnosed with pituitary adenoma, who underwent MTHS and whose preoperative nasal examinations were recorded between January 2007 and January 2014, were included in the study. Anterior rhinoscopic and endoscopic examinations were conducted on the patients and the presence of nasal septal perforations, nasal synechiae, purulent secretion into the nasal cavity, perinasal loss of sense, deflection of the nasal tip, a saddle nose deformity, hyposmia, anosmia, or an oronasal fistula was recorded by an ear nose and throat (ENT) specialist (I.E.). Nasal synechiae in the left and right nasal cavities were checked separately. The cases were classified based on synechiae between the septum and inferior or middle concha. Septal perforations classified by their location in the cartilaginous part were called anterior perforations, those located at the osseo-cartilaginous junction were called middle perforations, and perforations in the osseous part were called posterior perforations. Septal perforations classified by size were small $(<1 \mathrm{~cm})$, medium $(1-2 \mathrm{~cm})$, or large perforations $(>2 \mathrm{~cm})$.

\section{Surgical Technique}

The cartilaginous septum was incised approximately $2 \mathrm{~cm}$ posterior to the columella by entering the right nostril using a Killian type nasal retractor. This is the standard transsphenoidal approach to access the sphenoid sinus: after passing the subperichondrial plane and accessing the septal bone, a mucosal tunnel, which extended over the rostrum sphenoidale and the anterior wall of the sphenoid sinus, was driven in the subperiosteal plane. The cartilaginous septum was resected, and a retractor was placed in the anterior wall of the sphenoid sinus. After resecting the tumor and closing the sphenoid sinus, the Papavero type nasal retractor was removed, and the nasal septal mucosa was put in place. Merocel and a perforated internal nasal splint were placed in the nasal cavity bilaterally, and the operation was finished. Nasal packing was removed 2 days later.

\section{RESULTS}

In total, 14 patients were male and 17 were female. The mean ages of the female and male patients were $46.8 \pm 9.7$ and 49.6 \pm 14.6 years, respectively, and the overall mean age was 48 \pm 7 years.

The mean postoperative follow-up duration was $34.9 \pm 19.35$ months (range: 8 months to 7 years). Rhinological changes were found in 22 patients $(71 \%)$, but no rhinological pathology was found in 9 of the 31 patients.

The nasal septum was perforated in 12 of the 31 patients (38.7\%). The perforation in one of these patients spread almost throughout the entire septum, and a saddle nose deformity was observed. Another patient had two perforations; a small one was located in the anterior part and a middle-sized one was located in the posterior part. Two patients had small perforations, one had a middle-sized perforation, and seven had large-sized perforations. The most frequent location for a perforation was the middle part of the septum $(n=9)$. Perforations were found in the anterior part three times and in the posterior part three times (Figure 1).

No synechiae were detected between the nasal septum and the middle of the concha in the left nasal cavity, whereas two patients had synechiae between the nasal septum and the middle of the concha in the right nasal cavity. Seven patients had synechiae between the septum and inferior concha in the left nasal cavity, and five patients had synechiae between the septum and the inferior concha in the right nasal cavity (Figure 2). One patient had synechiae in both nasal cavities (between the septum and inferior concha in both nasal cavities). A nasal cavity synechiae was found in 13 of the 31 patients $(42 \%)$ (Figures 3-5).

Three patients had anosmia, two had hyposmia, and one patient with a nasal septum perforation had a nasal tip

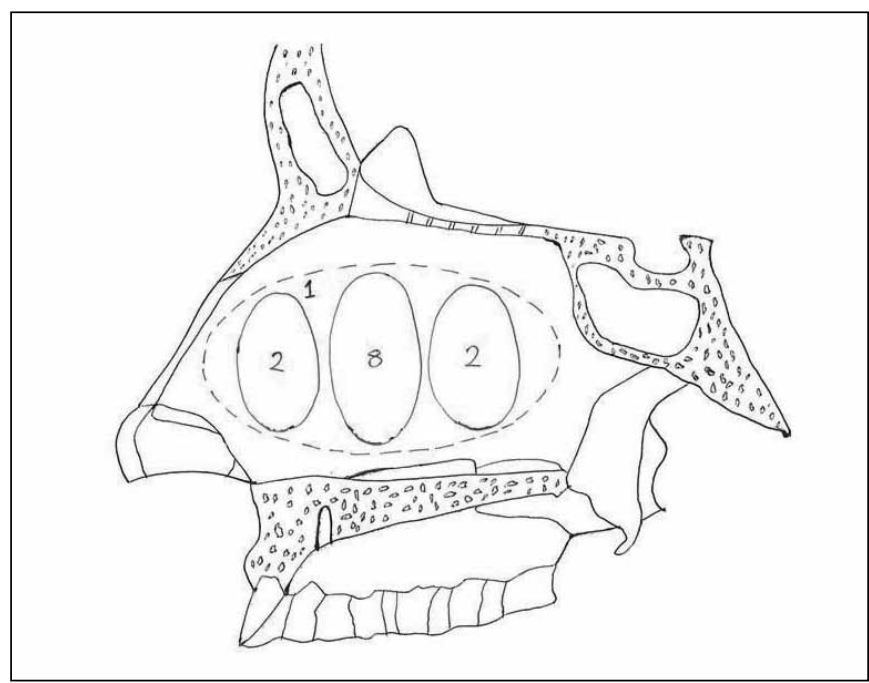

Figure 1: Localization of the nasal septum perforations.

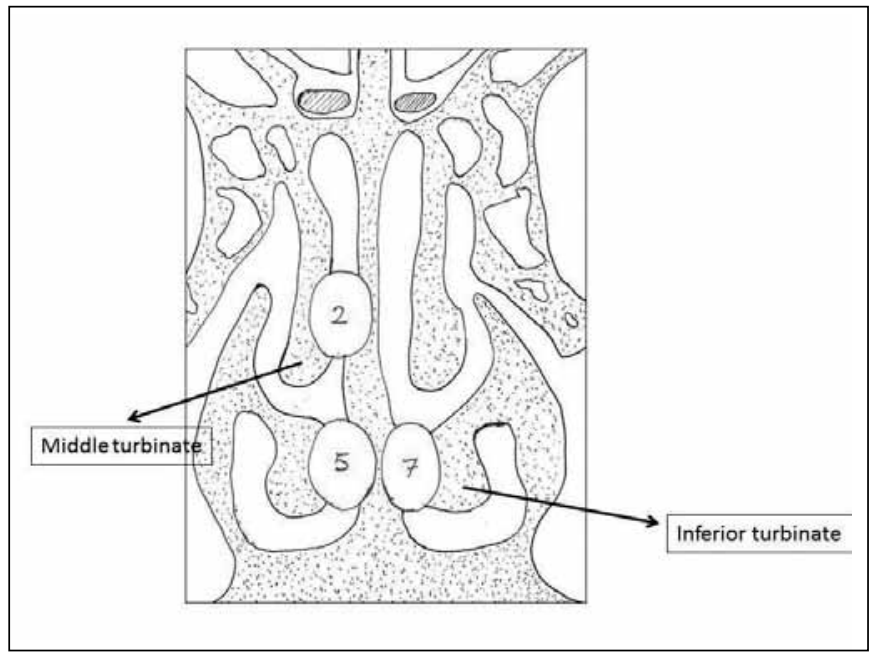

Figure 2: Localization of the nasal synechiae. 
deflection and a saddle nose deformity. No complications, such as perinasal loss of sense, oronasal fistula, or purulent secretion were found in the nasal cavities of any of the patients. Synechiae between the middle concha and septum in the right nasal cavity, and a large perforation in the middle part of the septum, were found in one patient with anosmia, whereas no synechiae or perforations were found in two patients with anosmia. A synechiae was found between the septum and inferior concha in the right nasal cavity in one of the patients with hyposmia, whereas no synechiae or perforations was found in the other patient.

Olfaction was normal during the preoperative period in patients with hyposmia and anosmia. Because hyposmia in two patients and anosmia in one patient, which occurred during the preoperative period, continued into the postoperative period, these patients were not included in the group with olfactory disorders.

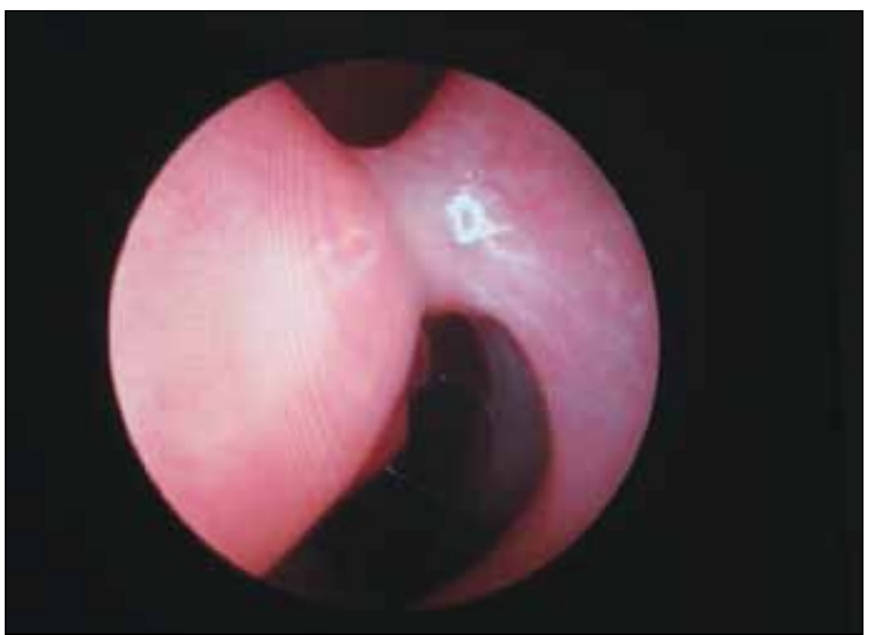

Figure 3: Synechiae between the inferior turbinate and nasal septum in the right nasal cavity.

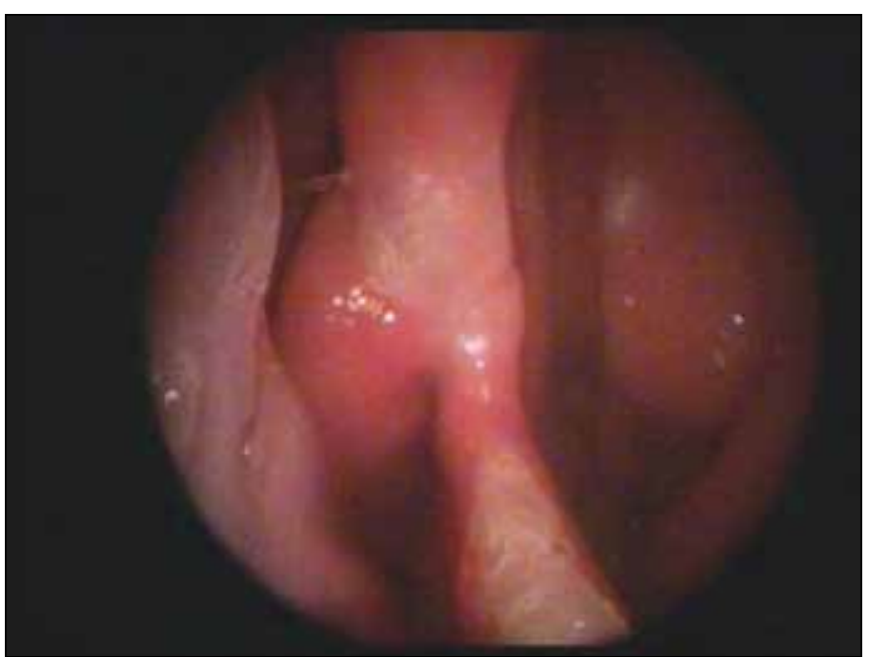

Figure 4: Septal perforation on location of the median nasal septum and synechiae between the middle turbinate and nasal septum.

\section{DISCUSSION}

Rhinological complications after MTHS can affect patient activities of daily living and quality of life. The reported incidence of rhinological complications associated with MTHS is $1.3-87.7 \%(2,11,17,20)$. In our study, the incidence of rhinological complications was $71 \%$. This wide range may be due to use of different parameters to determine the incidence rate. In some studies, the incidence of complications was very low because late postoperative complications, such as perinasal loss of sense, columellar retraction, or a saddle nose deformity, were not considered complications and only early postoperative complications were included in analyses (2). However, other studies considered findings and complaints, such as nasal crusting and irritation, as complications, which resulted in a higher incidence of complications (20). Considering the routine steps used by ENT specialists to perform a septoplasty, mitigating such complications would be useful.

Nasal septal perforations occur frequently as a result of bilateral mucosal lacerations in the septum. The symptoms are nasal obstruction, nose crusting, dry mucosa, intermittent epistaxis, nasal discharge, rhinorrhea, abnormal air flow, whistling sound during inspiration due to breathing through the nose, headache, and local pain. Small and anterior-type perforations often decrease humidity in the inspired air. Larger perforations may cause other nasal problems, such as atrophic rhinitis. A nasal septal perforation is an important problem that occurs after microscopic and endoscopic trans-sphenoidal surgery. A $2.1 \%$ incidence was reported in a study that used the microscopic approach, which was the lowest incidence in the literature (1). Septal perforations rates in endoscopic

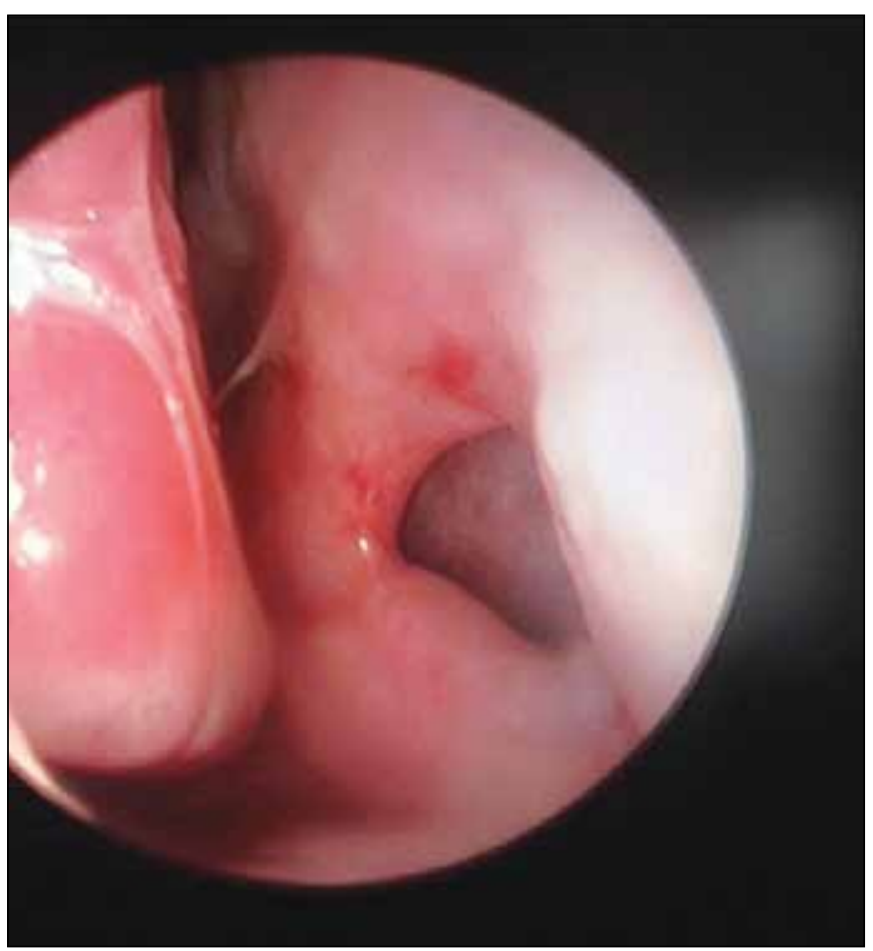

Figure 5: Perforation of the anterior nasal septum. 
series vary. However, no septal perforations were reported in two studies $(15,27)$, and perforation rates were $1.85-10 \%$ in three other studies $(12,14,24)$. Dew et al. reported an incidence of $18 \%(7)$. The highest incidence of septal perforation in the literature is $61 \%(20)$. In our study, 12 of 31 patients (38.7\%) had nasal septal perforations. Such different incidence rates in different studies may be associated with the experience of the surgeon or the technique used. The incidence of septal perforation was $7.6 \%$ in agroup of surgeons who had performed $<200$ trans-sphenoidal surgeries (TSS); however, it was 3.3\% in a group of surgeons who performed $>500$ TSS (5). Some studies have reported a $<1 \%$ incidence of septal perforations after septoplasty for a nasal septal deviation performed by ENT specialists. The highest reported percentage for similar groups is $6.7 \%(6,18,19,21,22,25)$. These data show the importance of experience and following the proper surgical steps. Nasal mucosal structure can be protected to prevent a septal perforation by using particular technical maneuvers. A submucosal injection of local anesthetic may help strip the nasal mucosa from the septum. Driving superior and inferior tunnels unilaterally, and then joining these tunnels and dislocating the nasal cartilage by approaching the other side through a posterior submucosal tunnel (thereby raising only one mucoperichondrial flap), is another protective maneuver. These maneuvers prevent bilateral lacerations of the mucosa and protect against the risk of a permanent septal defect. Any intraoperative perforations should be repaired immediately (5).

Synechiae, also called adhesions, are defined as inflamed bands of adjacent mucosa that cause postoperative nasal obstructions after septoplasty and other sinonasal operations. Synechiae usually occur between injured or abraded surfaces. We found only one study where postoperative synechiae after MTSS were examined, and a basal synechiae was found in 24 (48\%) of 49 patients (20). Only one endoscopic TSS study investigated synechiae, but no case was found (0\%) (27). In our study, synechiae were found in the nasal cavities of $13(42 \%)$ of the 31 patients. However, the incidence of this complication after septoplasty is approximately $7 \%$ (19). Controlling postoperative infection and minimizing intraoperative trauma are the best ways to avoid synechiae. Endonasal splints are also frequently used to stabilize the septum and prevent synechiae $(21,22)$.

We found no studies that discussed the locations of septal perforations and nasal synechiae. Our study is the first in which sizes of septal perforations were classified.

Olfactory receptors are located in the superior-medial part of the middle concha, the one-third most superior part of the septum, and the upper part of the superior concha in the nasal cavity. Intraoperative damage to the nasal olfactory system may cause an olfactory disorder, such as anosmia or hyposmia. Higgins et al. (12) reported a 2-12\% incidence rate for anosmia and hyposmia in patients who underwent TSS. Koren et al. (14) reported hyposmia and anosmia in two (10\%) of 20 patients who underwent a trans-nasal endoscopic operation. Tan and Jones (23) detected hyposmia in one (4\%) of 25 patients, and Actor et al. found olfactory deterioration in $35 \%$ of their patients (1). Charalampaki et al. (4) reported hyposmia and anosmia in 16 and 3 patients, respectively, of 200 patients who underwent endoscopic surgery for pituitary adenoma. Petry et al. detected hyposmia or anosmia in $10(20 \%)$ of 49 patients (20). The lowest incidence was $2 \%$, reported by White et al. in an endoscopic series (27). Hyposmia or anosmia occurs in approximately $1 \%$ of patients after septoplasty. Total rates of anosmia after a long-term septoplasty follow-up are $0.3-2.9 \%(21,22,25)$. We found olfactory disorders in 5 of 31 patients (16.2\%); 3 patients $(9.7 \%)$ had anosmia and $2(6.5 \%)$ had hyposmia. Synechiae were found between the inferior concha and septum in the right nasal cavity in one patient with hyposmia, and between the middle concha and septum in the right nasal cavity of one patient with anosmia. Our data suggest that olfactory disorders can occur regardless of the presence of synechiae in the nasal cavity. Surgeons must avoid using a laser to cut and avoid excessive electrocoagulation in the superior concha and superior part of the middle concha of the lateral nasal wall to overcome this complication, as this area contains a high concentration of olfactory nerve fibers. All kinds of mucosal damage occurring in these areas can cause olfactory disorders.

External nasal deformities may occur due to changes in the nasal skeleton after trans-nasal TSS. Petry et al. (20) reported a patient with right nasal valve stenosis accompanied by an external nasal deformity. Saddle nose is another type of external nasal deformity. A saddle nose deformity may occur as a consequence of excision, particularly when the superior part of the cartilaginous septum is cut, and stability of the septum is disrupted or there is an insufficient cartilage strut (5). In our study, a perforation in one patient spread over the entire septum, and a saddle nose deformity formed. The columellar tip strut in these patients may be poorer than normal and/or the incision can be made anteriorly.

Some postoperative sensorial disorders due to injuries to the anterior part of the palate and central incisors were reported in $2.8 \%$ of patients after septal surgery (3). These disorders usually occur due to injury to the nasopalatine nerve while gouging the maxillary crest. Therefore, the maxillary crest should be resected conservatively. A nasopalatine nerve injury-associated palatal sensorial disorder can occur after resecting the deviated part of the vomer and ethmoid bone (3). Petry et al. reported perinasal anesthesia after trans-nasal TSS in 2 of 49 patients (20). Varshney et al. detected numbness on the tip of the nose in one patient (1.85\%) (24). However, in our study, and in that of White et al. (27), no perinasal loss of sense was found in any patient. This may have resulted from keeping off the tabula where the anteroinferior part of the septum is located, and from the use of a Killian incision. Thus, injury to neural structures associated with the nasal septum, such as the superior alveolar nerve or nasopalatine nerve, was prevented.

We did not find any oronasal fistulas. However, Petry et al. found an oronasal fistula in 1 of 42 patients (20). We did not gouge the nasal base, which may have been why we did not encounter such a complication.

Postoperative sinusitis is another possible complication, with an incidence of $1-15 \%$ in microscopic TSS studies 
$(8,13,16,20)$. Two endoscopic TSS studies reported sinusitis rates of $1.75 \%$ and $8.5 \%(5,15)$. In our study, no purulent secretion was detected in the nasal cavities of any patient during the nasal endoscopic examinations. We did not examine the paranasal sinuses for sinusitis by computed tomography and did not observe sinusitis in any patient. Early removal of nasal packing and 7-10 days of postoperative oral antibiotic therapy may also have decreased the incidence of this complication (5).

\section{CONCLUSION}

Nasal structures, particularly the nasal septal mucosa, should be treated gently during MTHS. The operation should be performed in subperichondrial and subperiosteal planes to prevent a nasal septal perforation and all bilateral lacerations must be repaired. Nasal packing should be placed between the septum and inferior and middle conchae with care. The nasal stages of the operation should be performed with the help of an otolaryngologist until adequate experience is gained.

\section{- REFERENCES}

1. Actor B, Sarnthein J, Prömmel P, Holzmann D, Bernays RL: Olfactory improvement in acromegaly after transnasal transsphenoidal surgery. Neurosurg Focus 29(4):E10, 2010

2. Berker M, Hazer DB, Yucel T, Gurlek A, Cila A, Aldur M, Onerci M: Complications of endoscopic surgery of the pituitary adenomas: Analysis of 570 patients and review of the literature. Pituitary 15:288-300, 2012

3. Chandra RK, Rohman GT, Walsh WE: Anterior palate sensory impairment after septal surgery. Am J Rhinol 22(1):86-88, 2008

4. Charalampaki P, Ayyad A, Kockro RA, Perneczky A: Surgical complications after endoscopic transsphenoidal pituitary surgery. J Clin Neurosci16:786-789, 2009

5. Ciric I, Ragin A, Baumgartner C, Pierce D: Complications of transsphenoidal surgery: Results of a national survey, review of the literature, and personal experience. Neurosurgery 40(2):225-236, 1997

6. Daudia A, Alkhaddour U, Sithole J, Mortimore S: A prospective objective study of the cosmetic sequelae of nasal septal surgery. Acta Otolaryngol 126(11): 1201-1205, 2006

7. Dew LA, Haller JR, Major S: Transnasal transsphenoidal hypophysectomy: Choice of approach for the otolaryngologist. Otolaryngol Head Neck Surg 120(6):824-827, 1999

8. Faria MA Jr, Tindall GT: Transsphenoidal microsurgery for prolactin secreting pituitary adenomas. J Neurosurg 56(1):3343, 1982

9. Halsted AE: Remarks on the operative treatment of tumours of the hypophysis. With the report of two cases operated on by an oronasal method. Surg Gynecol Obstet 10:494-502, 1910

10. Hardy J: Transsphenoidal hypophysectomy. J Neurosurg 34:582-594, 1971
11. Hardy J: Surgery of the pituitary gland, using the open transsphenoidal approach: Comparative study of 2 technical methods. Ann Chir 21:1011-1022, 1967

12. Higgins TS, Courtemanche C, Karakla D, Strasnick B, Singh RV, Koen JL, Han JK: Analysis of transnasal endoscopic versus transseptal microscopic approach for excision of pituitary tumors. Am J Rhinol 22:649-652, 2008

13. Kennedy DW, Cohn ES, Papel ID, Holliday MJ: Transsphenoidal approach to the sella: The Johns Hopkins experience. Laryngoscope 94(8):1066-1074, 1984

14. Koren I, Hadar T, Rappaport ZH, Yaniv E: Endoscopic transnasal transsphenoidal microsurgery versus the sublabial approach for the treatment of pituitary tumors: Endonasal complications. Laryngoscope 109:1838-1840, 1999

15. Kumar S, Darr A, Hobbs CG, Carlin WV: Endoscopic, endonasal, transsphenoidal hypophysectomy: Retrospective analysis of 171 procedures. J Laryngol Otol 126:1033-1040, 2012

16. Mampalam TJ, Tyrrell JB, Wilson CB: Transsphenoidal microsurgery for Cushing disease. A report of 216 cases. Ann Intern Med 109(6):487-493, 1988

17. Monnier DS: Séquelles endonasales après hypophysectomie. Ann Otolaryngol Chir Cervicofac 115:49-53, 1998

18. Morre TD, Van Camp C, Clement PA: Results of the endonasal surgical closure of nasoseptal perforations. Acta Otorhinolaryngol Belg 49(3):263-267), 1995

19. Muhammad IA, Nabil-ur Rahman: Complications of the surgery for deviated nasal septum. J Coll Physicians Surg Pak 13(10):565-568, 2003

20. Petry C, Leães CGS, Pereira-Lima JFS, Gerhardt KD, Sant GD, Oliveira MC: Oronasal complications in patients after transsphenoidal hypophyseal surgery. Braz J Otorhinolaryngol 75(3):345-349, 2009

21. Rettinger G, Kirsche $\mathrm{H}$ : Complications in septoplasty. Facial Plast Surg 22(4):289-297, 2006

22. Schwab JA, Pirsig W: Complications of septal surgery. Facial Plast Surg 13(1):3-14, 1997

23. Tan LK, Jones RA: Nasal complications of the direct transnasal approach to the pituitary fossa. Br $\mathrm{J}$ Neurosurg 9:739-742, 1995

24. Varshney S, Gupta C, Bansal KK, Bist SS, Bhagat S: Endoscopic Trans-Nasal Trans-Sphenoidal (TNTS) approach for pituitary adenomas: Our experience. Indian J Otolaryngol Head Neck Surg 65(2):308-313, 2013

25. Vuyk HD, Langenhuijsen KJ: Aesthetic sequelae of septoplasty. Clin Otolaryngol Allied Sci 22(3):226-232, 1997

26. Welborn RB: The evolution of transsphenoidal pituitary microsurgery. Surgery 100:1185-1190, 1986

27. White DR, Sonnenburg RE, Ewend MG, Senior BA: Safety of minimally invasive pituitary surgery (MIPS) compared with a traditional approach. Laryngoscope, 114:1945-1948, 2004 